\title{
Roles of Fas/FasL and Complement Activation in Adult Patients With Chronic Active Epstein-Barr Virus Hepatitis
}

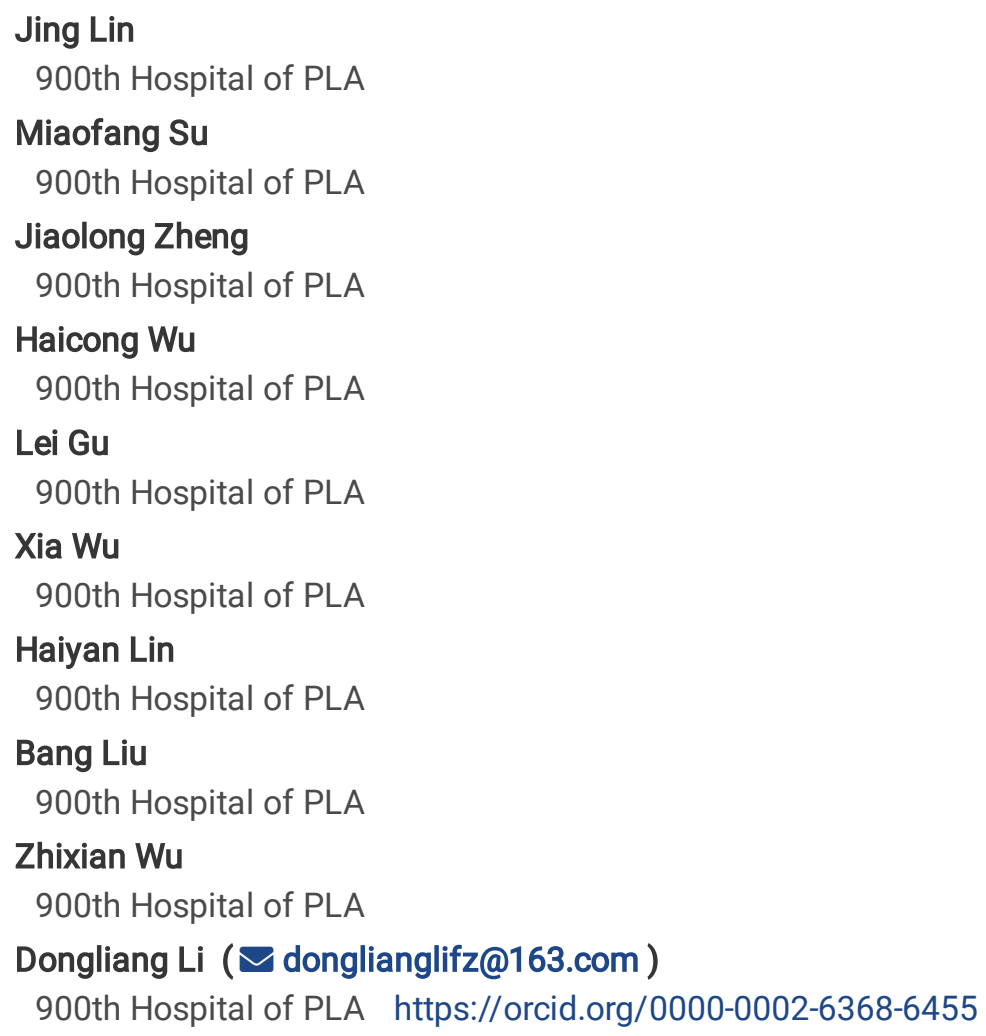

\section{Research}

Keywords: Chronic active EB virus hepatitis, Fas/FasL, Complement

Posted Date: October 19th, 2021

DOI: https://doi.org/10.21203/rs.3.rs-954937/v1

License: (c) (i) This work is licensed under a Creative Commons Attribution 4.0 International License. Read Full License 


\section{Abstract}

Background: Chronic active Epstein-Barr virus hepatitis (CAEBVH) in adult patients is a rare and highly lethal disease characterized by hepatitis and hepatomegaly.

Aims: To investigate the clinicopathological features and pathogenic mechanisms in patients with CAEBVH.

Methods:10 adult patients confirmed CAEBVH infection were collected. The clinicopathological characteristics were summarized and analyzed by clinical data. Flow cytometry to detect peripheral blood immune cell phenotypes, second-generation sequencing methods to explore pathogenic mechanisms, and immunohistochemical methods to verify pathogenic mechanisms.

Results: The clinical features included splenomegaly, hepatomegaly, abnormal liver function, and CD ${ }^{+} \mathrm{T}$ lymphopenia. HE also showed lymphocytic infiltration in liver tissue. EBER-ISH in lymphocytes of liver tissues were positive. Whole exon sequencing showed mutant genes were primarily enriched in 'T cell activation' and 'Complement and coagulation cascades'. The expression of $\mathrm{CD} 8$ in the CAEBVH group was higher than the controls in liver tissue $(p<0.05)$. The same as the expression of Fas, FasL, Caspase8 , and TUNEL assay $(p<0.05)$. Complement 1q (C1q) of liver sinusoidal endothelial cells (LSECs) and Glisson's capsule (GC), as well as Complement 3d (C3d) of LSECs, were a higher expression in CAEBV infection than controls $(p<0.05)$.

Conclusion: Fas/FasL and complement activation were involved in adult patients with chronic active Epstein-Barr virus hepatitis.

\section{Introduction}

In 1964, Epstein and Barr discovered a virus in the lymphocytes of children with Burkitt's lymphoma in Africa, which later became known as EBV. EBV is herpes virus type 4, a double-stranded DNA virus, complete with viral particles consisting of a nucleus, a membrane shell, shell particles and an envelope. EBV is one of the most common viruses in humans, affecting more than $90 \%$ adult population. ${ }^{1}$ Most infected individuals are asymptomatic, but adolescents often present with infectious mononucleosis (IM), which is a self-limiting disease with fever, pharyngitis, lymphadenopathy, hepatomegaly and splenomegaly. In rare cases of EBV infection, the above symptoms may persist and recur and are referred to as CAEBV infection. It is a rare and highly lethal disease characterized by multisystem inflammation with abnormally elevated EBV-associated antibodies in the blood and EBV-encode small RNA (EBER) in tissues. ${ }^{2}$ Adult patients with CAEBV infection are rare, have atypical symptoms, and have poor prognoses compared to children. Therefore, the disease has attracted great attention in recent years and related research reports are gradually increasing. ${ }^{3,4}$ However, it is primarily a view of the systemic multisystem damage. ${ }^{5}$ In contrast, little research has been reported on the clinical, pathological and injury mechanisms of CAEBVH. In this paper, 10 adults cases of CAEBVH diagnosed by liver biopsy and laboratory tests were included as study subjects. Analysis of the clinical and pathological features, and research of hepatitis mechanism in adult CAEBVH patients.

\section{Materials And Methods}

\subsection{Participant selection and data collection}

Patients with adult-onset CAEBV infection were defined as those whose estimated age at onset was 14 years or older and who met the criteria for the diagnosis of systemic EBV-T-LPD according to the 2016 World Health Organization (WHO) classifications of lymphoid neoplasms. ${ }^{5}$ (i) sustained or recurrent infectious mononucleosis-like symptoms lasting more than three months, including fever ( $\geq 38.3^{\circ} \mathrm{C}$ or $\geq 101^{\circ} \mathrm{F}$ ), liver dysfunction (elevated liver enzymes), lymphadenopathy, hepatosplenomegaly, cytopenia, interstitial pneumonia, hydroa vacciniforme, and hypersensitivity to mosquito bites; (ii) increased quantities of EBV in affected tissues [i.e., detection of EBV-DNA in tissues or peripheral blood by Southern blot hybridization or EB-encoded small RNA 1(EBER)-positive cells detected in affected tissues by microscopy ( $\geq 10$ cells/high power field)] or in peripheral blood [i.e., EBV-DNA detected in plasma ( $\geq 2 \times 10^{2}$ copies/mL in plasma)]; and (iii) no evidence of any previous immunological abnormalities or any other infections that could otherwise explain the condition. HLH was diagnosed according to HLH 2004 guidelines. ${ }^{6} 10$ patients diagnosed with CAEBV infection were included. $2 \mathrm{ml}$ of whole blood was collected intravenously from each patient and stored frozen at $-80^{\circ} \mathrm{C}$. Another 4 patients diagnosed with hemangioma and undergoing liver resection were included as a control group,

Page 2/15 
excluding liver damage caused by viruses, drugs, autoimmune diseases, etc. The clinicopathologic information (such as age, sex, laboratory examination, radiologic findings, therapeutic regimen and follow-up data) and liver samples were collected from the 900th Hospital of the Joint Logistic Support Force during the last eight years, from January 1, 2011, to December 30, 2019. Followup started on the day of diagnosis about CAEBV infection, and the end of follow-up was December 30, 2019, the date of death or the date of loss to follow-up. Informed consent was obtained from each patient included in the study. The study protocol conforms to the ethical guidelines of the 1975 Declaration of Helsinki (6th revision, 2008) as reflected in a priori approval by the institution's human research committee. The institutional review board approved this study of the 900th Hospital of the Joint Logistic Support Force(Review number:2020023).

\subsection{Flow cytometry}

Peripheral blood mononuclear cells were stained with fluorochrome-conjugated mAbs for flow cytometric analysis. CD3/CD8/CD45/CD4 (BD Biosciences, San Jose, CA, BD Biosciences Cat\# 340499, RRID:AB_400472), AntiCD3/CD16 ${ }^{+}$CD56/CD45/CD19(BD Biosciences Cat\# 340500, RRID:AB_400473) were used. All flow cytometry analyses were performed on BD FACS Canto flow cytometer (BD Biosciences) using BD FACS Canto clinical software version 2.0 (BD Biosciences) or BD FACS Diva software version 8.0 (BD Biosciences). Instrument performance was checked daily using the setup and tracking application BD FACS 7-Color Setup Beads (BD Biosciences). The acquisition gates were restricted to lymphocyte gates based on morphological characteristics, and at least 50,000 lymphocytes were acquired and analyzed. The results were expressed as a percentage of the studied cell population.

\subsection{Next-Generation Sequencing Technology}

The genomes of 10 cases with eligible formalin-fixed and peripheral blood samples were sequenced using Illumina HiSeq 2500 instrument. The raw data were aligned and analyzed for the detection of insertions/deletions and single-nucleotide variants. Gene Ontology (GO ( http://wiki.geneontology.org /index.php/Immunologically_Important_Genes) was then used to select immuneassociated gene mutations. Gene ontology (GO) and Kyoto Encyclopedia of Genes and Genomes (KEGG) enrichment were used for the screened genes. DAVID (https://david.ncifcrf.gov/) was used to conduct GO functional analysis and KEGG pathway enrichment analysis. $p<0.05$ was set as the cut-off criterion.

\subsection{Histologic Assessment}

The biopsy specimen was fixed in 10\% formalin and embedded in paraffin after routine processing. $3 \sim 4 \mu \mathrm{m}$ histologic sections were stained with hematoxylin and eosin ( $\mathrm{HE}$ ) for microscopic examination. The score of inflammation and steatosis referred to the previous studies. ${ }^{7,8}$

\subsection{In-situ hybridization of EB-encoded small RNA 1(ISH-EBER)}

ISH was conducted on formalin-fixed paraffin sections with a digoxin-labeled oligonucleotide probe complementary to 2 EBER, EBER-1, and EBER-2 (TIB, Xiamen, China, Lot \# 20170020) and a sense probe (negative control) was then labeled with digoxigenin (DIG) using previously described methods. ${ }^{9}$

\subsection{Immunohistochemistry (IHC) and Terminal deoxynucleotidyl transferase-mediated dUTP Nick-End Labeling( TUNEL)}

IHC staining on paraffin sections was performed for CD3(ZSGB-Bio,Beijing, China,Cat\# ZM-0417,RRID:AB_2890105),CD4(ZSGBBio Cat\# ZM0418,RRID:AB_2890106),CD8(ZSGB-Bio Cat\# ZA-0508,RRID:AB_2890107), CD20(Maixin, Fuzhou, China, Cat\# MAB0669, RRID:AB_2890108), CD56(ZSGB-Bio Cat\# ZM-0057, RRID:AB_2890109), perforin(Maixin Cat\# MAB-0315, RRID:AB_2890110), GrB(Maixin Cat\# MAB-0352, RRID:AB_2890111), Fas(Abcam,Cambridge, MA, USA, Cat\# ab82419, RRID:AB_1658628), FasL(Abcam Cat\# ab10041, RRID:AB_296781), caspase-8(Abcam Cat\#ab25901,RRID:AB_448890), C1q(Abcam Cat\# ab11861, RRID:AB_298643), C3d(Abcam Cat\# ab17453, RRID:AB_443879) and C4d(Abcam Cat\# ab36075, RRID:AB_726920). The IHC staining was performed as previously described. ${ }^{10}$ MageJ's quantitative evaluation and automatic scoring of IHC images. Hscore $=\sum(\mathrm{i} \otimes \mathrm{Pi})$, i represented staining intensity, graded as one of the following: 0, 1,2 or 3. Pi represents the percentage of stained cells. TUNEL staining was performed using the One-Step TUNEL Apoptosis Assay Kit (Dalian, China, MeilunBio Lot \# MA0223) to detect apoptosis of liver tissue. The method was performed as previously described. ${ }^{11}$ The apoptotic 
cells were characterized by positive fluorescence detection. One thousand cells were counted, the positive cells were identified, counted, and analyzed. The apoptotic index (Al) was expressed as the percentage of positive cells (Nikon 80i, Tokyo, Japan).

\subsection{Statistical analysis}

Data analysis was performed using GraphPad Prism (version 8.4.3, GraphPad Software, San Diego, CA). Measurement data with a normal distribution are expressed as the mean \pm SD. Measurement data with a nonnormal distribution are expressed as the median (interquartile spacing). Statistical analysis was performed using the Student's $t$-tests. $p<0.05$ was considered a statistically significant difference.

\section{Results}

\subsection{Clinical Features}

Of the 10 patients enrolled, 7 were male and 3 were female. The median age was $32(25.5,37.25)$ years (range $14 \sim 62)$. The most prominent symptom was fever(7/10). Radiologic findings showed splenomegaly (10/10) and hepatomegaly (9/10) (Fig. 1). Laboratory examination showed most patients elevated liver function indexes, manifested by mild to moderate abnormal of ALT or AST levels (9/10). The measured EBV load varied from $1.75 \times 10^{4}$ to $1.74 \times 10^{7} \mathrm{IU} / \mathrm{mL}$. Some patients had reduced CD $8^{+}$ lymphocyte counts (6/6) (Fig. 2). Inflammatory indicators such as Ferritin were elevated in our patients(5/7). But some of them have normal C-reactive protein (CRP) (5/9). Most patients received hepatoprotective, antiviral (ganciclovir) and immunosuppressive therapy (9/10). Immunosuppressive therapy included glucocorticoids, immunoglobulin combinations and chemotherapy drugs (cyclophosphamide, cyclosporine A, etoposide, vincristine, tacrolimus). Half of them died (5/10), with a median survival time of $3.0(1.5,4.0)$ months. More detailed information of all patients was shown in Table $1 \sim 2$.

Table 1

Clinical characteristics of patients with CAEBV infection

\begin{tabular}{|c|c|c|c|c|c|c|}
\hline Case & Gender/age & Symptoms & Imaging Findings & Treatment & Progress & Follow-up \\
\hline 1 & $M / 25$ & Fever & $\begin{array}{l}\text { Hepatomegaly and } \\
\text { splenomegaly }\end{array}$ & $\begin{array}{l}\text { Antiviral, immunosuppressive and } \\
\text { hepatoprotection }\end{array}$ & / & $\mathrm{D}(4.0)$ \\
\hline 2 & $\mathrm{M} / 14$ & / & $\begin{array}{l}\text { Hepatomegaly and } \\
\text { splenomegaly }\end{array}$ & $\begin{array}{l}\text { Antiviral, immunosuppressive and } \\
\text { hepatoprotection }\end{array}$ & / & $A(58.0)$ \\
\hline 3 & $M / 16$ & Fever & $\begin{array}{l}\text { Hepatomegaly and } \\
\text { splenomegaly }\end{array}$ & $\begin{array}{l}\text { Antiviral, immunosuppressive and } \\
\text { hepatoprotection }\end{array}$ & / & $\mathrm{D}(0.5)$ \\
\hline 4 & $M / 33$ & Fever & $\begin{array}{l}\text { Hepatomegaly and } \\
\text { splenomegaly }\end{array}$ & Antiviral and hepatoprotection & / & $\mathrm{A}(4.0)$ \\
\hline 5 & $M / 31$ & Fever & $\begin{array}{l}\text { Hepatomegaly and } \\
\text { splenomegaly }\end{array}$ & $\begin{array}{l}\text { Antiviral, immunosuppressive and } \\
\text { hepatoprotection }\end{array}$ & $\mathrm{HLH}$ & $\mathrm{D}(1.5)$ \\
\hline 6 & $\mathrm{~F} / 52$ & Fever & $\begin{array}{l}\text { Hepatomegaly and } \\
\text { splenomegaly }\end{array}$ & $\begin{array}{l}\text { Antiviral, immunosuppressive and } \\
\text { hepatoprotection }\end{array}$ & $\mathrm{HLH}$ & $\mathrm{D}(3.0)$ \\
\hline 7 & $F / 27$ & Fever & $\begin{array}{l}\text { Hepatomegaly and } \\
\text { splenomegaly }\end{array}$ & / & / & $A(14.0)$ \\
\hline 8 & $M / 38$ & Fever & $\begin{array}{l}\text { Hepatomegaly and } \\
\text { splenomegaly }\end{array}$ & $\begin{array}{l}\text { Antiviral, immunosuppressive and } \\
\text { hepatoprotection }\end{array}$ & $\mathrm{HLH}$ & $\mathrm{D}(12.0)$ \\
\hline 9 & $M / 35$ & / & $\begin{array}{l}\text { Hepatomegaly and } \\
\text { splenomegaly }\end{array}$ & $\begin{array}{l}\text { Antiviral, immunosuppressive and } \\
\text { hepatoprotection }\end{array}$ & $\mathrm{HLH}$ & $\mathrm{A} / \mathrm{R}(15.0)$ \\
\hline 10 & $\mathrm{~F} / 62$ & / & Splenomegaly & Antiviral and hepatoprotection & / & $A(6.5)$ \\
\hline
\end{tabular}


Table 2

Clinical characteristics of patients with CAEBV infection

\begin{tabular}{|c|c|c|c|c|c|c|c|c|c|c|c|c|}
\hline \multirow[t]{3}{*}{ Case } & \multicolumn{6}{|c|}{ Blood lymphocytes analysis } & \multicolumn{3}{|c|}{ Liver function } & \multirow{2}{*}{$\begin{array}{l}\text { EBV } \\
\text { DNA }\end{array}$} & \multicolumn{2}{|c|}{ Inflammation } \\
\hline & CD3 & $\mathrm{CD} 4$ & CD8 & CD16+56 & CD19 & CD4/ & ALT & AST & TBIL & & Ferritin & CRP \\
\hline & $(\%)$ & $(\%)$ & $(\%)$ & $(\%)$ & $(\%)$ & CD8 & $(U / L)$ & $(U / L)$ & (umol/L) & (IU/mL) & $\mathrm{ng} / \mathrm{mL}$ & $\mathrm{ug} / \mathrm{mL}$ \\
\hline 1 & 95 & 28.7 & 16.9 & / & / & 1.7 & 127 & 313 & 198.7 & $1.23 \times 10^{6}$ & 118 & $\llbracket 3.3$ \\
\hline 2 & 25.13 & 16.93 & 7.04 & 53.44 & 19.92 & 2.41 & 47 & 238 & 143.6 & $4.10 \times 10^{5}$ & 52.5 & $\varangle 3.3$ \\
\hline 3 & 26.91 & 20.26 & 5.8 & / & / & 0.29 & 80.8 & 125.2 & 151.2 & $1.47 \times 10^{7}$ & / & 2.08 \\
\hline 4 & 66.61 & 38.65 & 15.26 & 18.81 & 11.46 & 2.53 & 83.1 & 57 & 20.6 & $6.83 \times 10^{6}$ & I & / \\
\hline 5 & I & / & I & I & / & / & 136.1 & 234.3 & 60.2 & $2.43 \times 10^{5}$ & $\otimes 1888$ & 100 \\
\hline 6 & I & I & I & 6.6 & I & / & 21.8 & 47.4 & 5.6 & $1.15 \times 10^{5}$ & 1102 & 17.88 \\
\hline 7 & 47.4 & 29.3 & 16.9 & 49 & / & 1.8 & 5.8 & 26.5 & 8.5 & $1.99 \times 10^{6}$ & I & $\otimes 7.2$ \\
\hline 8 & 16.9 & 7.5 & 8.6 & 80.5 & 2.1 & 0.87 & 73.9 & 43 & 9.7 & $1.02 \times 10^{7}$ & 1129 & 15.6 \\
\hline 9 & l & I & I & I & I & I & 95.5 & 43.1 & 15.7 & $1.75 \times 10^{4}$ & 1502 & $\otimes 7.2$ \\
\hline 10 & l & I & / & / & I & / & 93.3 & 151.3 & 57.5 & $2.30 \times 10^{6}$ & 322.5 & 11.8 \\
\hline
\end{tabular}

\subsection{Histologic Features and target cells}

The liver is one of the most common target organs, presenting with liver injury and hepatomegaly. Normal liver tissue from patients aged diagnosed with hemangioma was included as the control group. There was no statistical difference between the two groups regarding gender and age $(p>0.05)$. HE showed varying degrees of steatosis, edema and inflammatory cell infiltration, companying a small amount of hepatocyte necrosis in the liver tissue of the group with CAEBV infection. The score of inflammation and steatosis in the group with CAEBV infection was higher than in the control group $(p<0.05)$.

EBER-ISH of liver tissues showed that lymphocytes, not NK cells, were positive in the CAEBV infection group, which clarified that T cells were target cells of EBV infection (Fig. 2).

\subsection{Next-Generation Sequencing}

Because CAEBV infection is a systemic disease rather than solid tumors with abnormal genetic mutations in the tissue, peripheral blood cell immune genes can reflect on systemic immunity. Besides, CAEBVH is extremely rare in clinical practice, and liver puncture biopsy is an invasive test. So we detected mutations in host immune genes of peripheral blood cells using whole-exome sequencing to explore pathogenic mechanisms of CAEBV infection.

A total of 410 immune genes were screened in 10 patients. GO describes genes from three aspects, namely molecular function (MF), cellular component (CC) and biological process (BP). In the BP group, mutant genes were primarily enriched in 'T cell activation', 'regulation of immune effector process' and 'leukocyte proliferation. In the CC group, mutant genes were primarily enriched in the 'external side of plasma membrane', 'secretory granule membrane' and 'endocytic vesicle'. In the MF group, mutant genes were primarily enriched in 'immune receptor activity', 'cytokine receptor binding' and 'amide binding '. KEGG pathway enrichment analysis demonstrated that the mutant genes were primarily associated with 'Human papillomavirus infection', 'Complement and coagulation cascades' and 'Cytokine-cytokine receptor interaction' (Fig. 3).

\subsection{Mechanisms of CAEBVH}


Based on the GO enrichment results, lymphocyte phenotypes were detected to explore whether $\mathrm{T}$ lymphocyte activation was involved in the CAEBV infection. The expression of $\mathrm{CD} 8^{+}$in the CAEBVH group was higher than the control group $(p<0.05)$. However, there were no significant differences about CD3, CD4, CD56 and CD20 in the two groups ( $p>0.05)$ (Fig. 4). To further investigate the mechanism of immune damage in $C D 8^{+} T$ lymphocytes, we examined the expression of perforin, granzyme $B$, Fas,Fas and caspase-8. The expression of perforin and granzyme B was not different between the two groups $(p>0.05)$. However, the expression of Fas, FasL and caspase-8 were higher in the CAEBVH group $(p<0.05)$ (Fig. 5A).TUNEL assay also showed that apoptosis index (Al) was higher in the CAEBVH group ( $p<0.05)$ (Fig. 5B).

Based on the KEGG enrichment results, we investigated whether the complement pathway is involved in the pathogenesis of CAEBVH. We detected expression levels of C1q, C3d and C4d, an essential component of the complement pathway in LSECs and the GC of liver tissues. Since the liver is an immune organ, the C3d could be oddly deposited in $\mathrm{GC} .{ }^{12}$ But there was no deposition of $\mathrm{C} 1 \mathrm{q}$ and $\mathrm{C} 4 \mathrm{~d}$ in the normal liver tissue. ${ }^{13,14}$ The expression of C1q in LSEC and GC and C3d in LSECs were higher in the CAEBVH group $(p<0.05)$. The expression of C4d in LSECs and GC and C3d in GC showed no significant difference between the two groups ( $p>0.05$ ) (Fig. 6).

\section{Discussion}

CAEBV infection is characterized by clonal proliferation of EBV-infected T cells or NK cells in East Asian countries. It is categorized as an EBV-positive T/ NK cell tumor in the 2016 revision of the WHO classification of hematopoietic and lymphoid tissue tumors, generating widespread interest. The pathogenesis of CAEBV infection is still unclear, and it has been suggested that two factors may be present: the characteristics of the virus and the immune deficiency of the host. ${ }^{15}$ Although EBV can contribute to the development of CAEBV infection by expressing various proteins that interfere with the secretion of cytokines and inhibit apoptosis of infected cells, the prevalence of EBV infection in the population is high. In contrast, the incidence of CAEBV infection is low. Thus, a specific EBV strain may cause CAEBV infection with high virulence or a unique immune escape mechanism. Studies have identified EBV strains with high lytic replication rates. ${ }^{16,17}$ Yet, no CAEBV infection outbreaks or household transmission have been detected to date, suggesting that host immune factors rather than viral factors may play a key role in CAEBV infection.

CAEBVH is an essential clinical phenotype of CAEBV infection. Clinical observation found that patients of CAEBVH had more apparent symptoms, faster progression, and poorer prognosis than patients without liver damage. ${ }^{18}$ Therefore, this study analyzed the clinicopathological characteristics and investigated the mechanism of CAEBVH.

In our study, clinical features mainly included abnormal liver function (9/10), hepatomegaly (9/10), fever (7/10), splenomegaly (10/10), and elevated EBV-DNA viral load. This was mainly following T cell type, characterized by high fever, enlarged liver or spleen or lymph nodes, and EBV antibody-specific change. As we know, CAEBV infection can understand inflammatory diseases that lead to $\mathrm{HLH}$, and ferritin is an indicator. Ferritin is closely related to the progression and regression of disease conditions. Some patients had elevated ferritin (5/7) and progressed to HLH (4/7) in our study. In our study, 4 patients who had HLH with high ferritin died or recurred.

There are three main features in the liver histopathological examination. One is that inflammatory changes with steatosis in patients with CAEBVH. Secondly, the inflammatory cells, mainly lymphocytes, infiltrated the confluent area and hepatic sinusoids. Thirdly, EBER-ISH was positive in these lymphocytes. It was shown not EBV but lymphocytes caused liver damage in CAEBVH, unlike viral hepatitis, B. And consistent with clinical features, T cells are the target cells of EBV infection.

Immune cell phenotyping showed decreased $\mathrm{CD} 8^{+} \mathrm{T}$ lymphocytes, suggesting that $\mathrm{CD} 8^{+} \mathrm{T}$ lymphocytes may play an essential role in CAEBV infection. To explore the definite mechanism of CAEBVH, we detected mutations in host immune genes using wholeexome sequencing. Our study showed mutant genes were primarily enriched in ' $T$ cell activation'. We further found more $C D 8^{+} T$ lymphocytes in the CAEBV infection group than in the control group. However, no difference between $C D 3^{+} T, C D 4^{+} T, C D 20^{+} B$ lymphocytes, and $\mathrm{CD}_{56}{ }^{+} \mathrm{NK}$ cells. In the present research, reduced number and impaired function of EBV-specific CD $8^{+} \mathrm{T}$ lymphocytes were also found in CAEBV hosts. ${ }^{19,20}$ 
CD8 ${ }^{+} \mathrm{T}$ lymphocytes are essential immune cells in the body and play a critical role in fighting virus infection in the host. ${ }^{21}$ It was reported that the perforin/granzyme $B$ and the Fas/FasL apoptosis played a vital role in CD8 ${ }^{+} T$ lymphocytes. ${ }^{22-25}$ The TUNEL assay showed distinct apoptosis in the CAEBVH group than controls in our study. Perforin and granzyme B induced target cell death via non-caspase-dependent apoptotic pathway. ${ }^{26}$ And it was found perforin gene mutations in a patient with CAEBV infection. ${ }^{27}$ But our study found the expression of granzyme B and perforin had no significant difference between the two groups, and there were no perforin gene mutations. Hence, perforin and granzyme pathways need to be furth confirmed.

We further investigated the role of the caspase-dependent apoptotic pathway in CAEBVH. It was showed that Fas, FasL and caspase-8 were highly expressed in the CAEBVH group. Nomura et al. ${ }^{28}$ found caspase-3, critical apoptosis pathway proteins, highly expressed in CAEBV patients. When Fas of lymphocytes combined with FasL of tissue cells, activating caspase-8 of tissue cells to conduct caspase-dependent apoptosis pathway. ${ }^{29,30}$ In our study, high expression of caspase-8 in lymphocytes suggested lymphocytes were undergone excessive apoptosis due to abnormal proliferation. ${ }^{31,32}$ Activated caspase-8 also promoted the secretion of the inflammatory cytokine to cause further tissue damage. ${ }^{33}$ Keiko Nomura et al. ${ }^{34}$ had identified three Japanese patients with CAEBVH who had original Fas pathway-associated mutations. Thus, gene mutations associated with the Fas/FasL pathway may lead to CAEBVH; however, whether the mutations were primary or secondary need further studies.

KEGG enrichment suggested the complement activation may be a possible mechanism of CAEBVH. $\mathrm{C} 3$ can activate $\mathrm{CD} 8^{+} \mathrm{T}$ cells and promote proliferation. ${ }^{35} \mathrm{C} 1 \mathrm{q}$ was activated when it was bound to the immune complex and subsequently initiated the classical complement pathway. The classical pathway and lectin pathway activated C4d. And C3d was engaged in the three pathways, including classical, lectin and alternative pathways. ${ }^{36}$ Our study found the expression of C1q in LSECs and GC was higher in the CAEBVH group than in the controls. C3d in the LSECs was higher in the CAEBVH group. No C4d expression was observed in either group, considering that the alternative pathway, not the lectin and classical pathway, might be an essential pathogenic mechanism of CAEBV infection. Owing to C4d is a marker of B-lymphocyte immune response, such as immune complexes and antibodies. It was reflected the T-lymphocyte rather than the B-lymphocyte involved in the mechanism of CAEBVH. The imbalance of C3-derived fragments was the key to autoimmune and neoplastic diseases. ${ }^{37,38}$ Therefore, we inferred that the complement pathway could be involved in CAEBVH, which might help drug development efforts.

In summary, we hypothesize that EBV infection of lymphocytes leads to Fas/FasL and complement activation in CAEBH, which causes a storm of inflammatory cytokine and neoplastic diseases. Therefore, CAEBV infection treatment requires control of inflammation and immune cell proliferation. Standard therapies are antiviral and immunosuppression. Although patients $(5 / 10)$ received the antiviral and immunosuppressive treatment, they still died with a median survival time of $3.0(1.5,4.0)$ months in our study. Some reports showed that allogeneic hematopoietic stem cell transplantation (allo-HSCT) could cure CAEBVH ${ }^{39}$. In other words, immune replacement and reconstitution played a vital role in CAEBVH. It is implied gene mutations in host may be the essential reason. Therefore, targeted gene therapy may have implications for clinical treatment.

\section{Conclusion}

CAEBVH is extremely rare in clinical practice, Fas/FasL and complement activation were involved in adult patients. Tere were some shortcomings in this study: liver puncture biopsy is an invasive test, resulting in a relatively small number of subjects for this study; there is still a need to accumulate and expand the sample size and improve the methods of the corresponding tests for indepth research in the future.

\section{Declarations}

Acknowledgments: The authors thank the patients and their families for agreeing to use their data and specimens for research purposes, specifically for the publication this report.

Authors' Contributions: Jing Lin participated in manuscript drafting. Jing Lin and Miaofang Su completed the experiment. Jiaolong Zheng collected and analyzed clinical data. Xia Wu, Haicong Wu, Lei Gu, Haiyan Lin and Bang Liu checked the article 
quotations' smoothness, corrected the misspelled words, and organized literature. Dongliang Li participated in data interpretation and manuscript revision. All authors read and approved the final manuscript.

Funding: This work was supported by the Startup Fund for scientific research, Fujian Medical University (Grant Number: 2019QH1285), The 900th Hospital of the Joint Logistic Support Force Fund (Grant Number: 2018J07), Natural Science Foundation of Fujian Province of China (Grant Number: 2020J011131) and Natural Science Foundation of Fujian Province of China (Grant Number: 2021J011263).

Data availability statement: The data used for this study are not freely available because of human participants. Interested researchers can contact the corresponding authors through email for more detailed information.

Ethics approval and consent to participate: This study was conducted in accordance with the Declaration of Helsinki and approved by the Institutional Review Board (IRB) of the 900th Hospital of the Joint Logistic Support Force(Review number:2020023).

Consent for publication: All authors have read and approved the fnal manuscript. All parents signed informed consent forms and approved the fnal manuscript.

Competing interests: The authors declare that they have no competing interests.

\section{References}

1. Bunchorntavakul C, Reddy KR. Epstein-Barr Virus and Cytomegalovirus Infections of the Liver. Gastroenterol Clin North Am. 2020;49(2):331-46.

2. Abe N, Fujieda Y. Chronic active Epstein-Barr virus infection. Blood. 2020;136(18):2090.

3. Latour S, Fischer A. Signaling pathways involved in the T-cell-mediated immunity against Epstein-Barr virus: Lessons from genetic diseases. Immunol Rev. 2019;291(1):174-89.

4. Arai A. Advances in the Study of Chronic Active Epstein-Barr Virus Infection: Clinical Features Under the 2016 WHO Classification and Mechanisms of Development. Front Pediatr. 2019;7:14.

5. Lin J, Wu H, Gu L, et al. Clinicopathologic findings of chronic active Epstein-Barr virus infection in adults: A single-center retrospective study in China. Clin Exp Med. 2021.

6. La Rosée P, Horne A, Hines M, et al. Recommendations for the management of hemophagocytic lymphohistiocytosis in adults. Blood. 2019;133(23):2465-77.

7. Kleiner DE, Brunt EM, Van Natta M, et al. Design and validation of a histological scoring system for nonalcoholic fatty liver disease. Hepatology (Baltimore Md). 2005;41(6):1313-21.

8. Bedossa P. Pathology of non-alcoholic fatty liver disease. Liver Int. 2017;37(Suppl 1):85-9.

9. Weiss LM, Jaffe ES, Liu XF, Chen YY, Shibata D, Medeiros LJ. Detection and localization of Epstein-Barr viral genomes in angioimmunoblastic lymphadenopathy and angioimmunoblastic lymphadenopathy-like lymphoma. Blood. 1992;79(7):178995.

10. Gao L-M, Zhao S, Liu W-P, et al. Clinicopathologic Characterization of Aggressive Natural Killer Cell Leukemia Involving Different Tissue Sites. Am J Surg Pathol. 2016;40(6):836-46.

11. Yu X-Y, Chen H-M, Liang J-L, et al. Hyperglycemic myocardial damage is mediated by proinflammatory cytokine: macrophage migration inhibitory factor. PloS one. 2011;6(1):e16239.

12. Huang HJ, Feng CY, Zheng ZY. [Expression of C3d in normal human liver tissues with non-immunologic osmostic mechanism]. Zhonghua Bing Li Xue Za Zhi. 2017;46(9):629-33.

13. Bouron-Dal Soglio D, Rougemont A-L, Herzog D, Soucy G, Alvarez F, Fournet J-C. An immunohistochemical evaluation of C4d deposition in pediatric inflammatory liver diseases. Human pathology. 2008;39(7):1103-10.

14. Thorgersen EB, Barratt-Due A, Haugaa $\mathrm{H}$, et al. The Role of Complement in Liver Injury, Regeneration, and Transplantation. Hepatology (Baltimore Md). 2019;70(2):725-36. 
15. Arai A. Advances in the Study of Chronic Active Epstein-Barr Virus Infection: Clinical Features Under the 2016 WHO Classification and Mechanisms of Development. Front Pediatr. 2019;7:14.

16. Alfieri C, Ghibu F, Joncas JH. Lytic, nontransforming Epstein-Barr virus (EBV) from a patient with chronic active EBV infection. Canadian Medical Association journal. 1984;131(10):1249-52.

17. Jäger M, Prang N, Mitterer $M$, et al. Pathogenesis of chronic Epstein-Barr virus infection: detection of a virus strain with a high rate of lytic replication. Br J Haematol. 1996;95(4):626-36.

18. Schechter S, Lamps L. Epstein-Barr Virus Hepatitis: A Review of Clinicopathologic Features and Differential Diagnosis. Arch Pathol Lab Med. 2018;142(10):1191-5.

19. Xing Y, Song HM, Wei M, Liu Y, Zhang YH, Gao L. Clinical significance of variations in levels of Epstein-Barr Virus (EBV) antigen and adaptive immune response during chronic active EBV infection in children. J Immunotoxicol. 2013;10(4):387-92.

20. Sugaya N, Kimura H, Hara S, et al. Quantitative analysis of Epstein-Barr virus (EBV)-specific CD8+ T cells in patients with chronic active EBV infection. J Infect Dis. 2004;190(5):985-8.

21. Dotti G, Savoldo B, Pule M, et al. Human cytotoxic T lymphocytes with reduced sensitivity to Fas-induced apoptosis. Blood. 2005;105(12):4677-84.

22. Ling GS, Crawford G, Buang N, et al. C1q restrains autoimmunity and viral infection by regulating CD8 T cell metabolism. Science (New York NY). 2018;360(6388):558-63.

23. Lee JY, Chae DW, Kim SM, et al. Expression of FasL and perforin/granzyme B mRNA in chronic hepatitis B virus infection. J Viral Hepat. 2004;11(2):130-5.

24. Feldmann G, Lamboley C, Moreau A, Bringuier A. Fas-mediated apoptosis of hepatic cells. Biomed Pharmacother. 1998;52(9):378-85.

25. Kim JH, Kang TH, Noh KH, et al. Enhancement of dendritic cell-based vaccine potency by anti-apoptotic siRNAs targeting key pro-apoptotic proteins in cytotoxic CD8(+) T cell-mediated cell death. Immunol Lett. 2009;122(1):58-67.

26. Voskoboinik I, Whisstock JC, Trapani JA. Perforin and granzymes: function, dysfunction and human pathology. Nat Rev Immunol. 2015;15(6):388-400.

27. Katano H, Ali MA, Patera AC, et al. Chronic active Epstein-Barr virus infection associated with mutations in perforin that impair its maturation. Blood. 2004;103(4):1244-52.

28. Nomura Y, Kimura H, Karube K, et al. Hepatocellular apoptosis associated with cytotoxic T/natural killer-cell infiltration in chronic active EBV infection. Pathol Int. 2009;59(7):438-42.

29. Cohen GM. Caspases: the executioners of apoptosis. The Biochemical journal. 1997;326 (Pt 1).

30. Zheng M, Karki R, Vogel P, Kanneganti T-D. Caspase-6 Is a Key Regulator of Innate Immunity, Inflammasome Activation, and Host Defense. Cell. 2020;181(3).

31. Sugaya N, Kimura H, Hara S, et al. Quantitative analysis of Epstein-Barr virus (EBV)-specific CD8+ T cells in patients with chronic active EBV infection. J Infect Dis. 2004;190(5):985-8.

32. Pérez-Garijo A. When dying is not the end: Apoptotic caspases as drivers of proliferation. Semin Cell Dev Biol. 2018;82:86-95.

33. Van Opdenbosch N, Lamkanfi M. Caspases in Cell Death, Inflammation, and Disease. Immunity. 2019;50(6):1352-64.

34. Nomura K, Kanegane $\mathrm{H}$, Otsubo K, et al. Autoimmune lymphoproliferative syndrome mimicking chronic active Epstein-Barr virus infection. Int J Hematol. 2011;93(6):760-4.

35. Kanekiyo M, Bu W, Joyce MG, et al. Rational Design of an Epstein-Barr Virus Vaccine Targeting the Receptor-Binding Site. Cell. 2015;162(5):1090-100.

36. Merle NS, Church SE, Fremeaux-Bacchi V, Roumenina LT. Complement System Part I - Molecular Mechanisms of Activation and Regulation. Front Immunol. 2015;6:262.

37. Reis ES, Mastellos DC, Hajishengallis G, Lambris JD. New insights into the immune functions of complement. Nat Rev Immunol. 2019;19(8):503-16.

38. Roumenina LT, Daugan MV, Petitprez F, Sautès-Fridman C, Fridman WH. Context-dependent roles of complement in cancer. Nat Rev Cancer. 2019;19(12):698-715. 


\section{Figures}

A

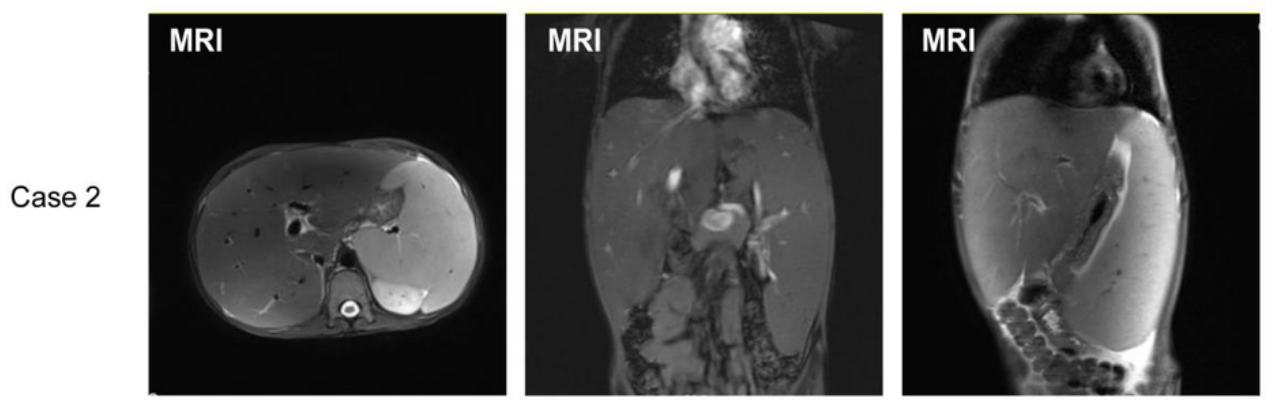

\section{B}
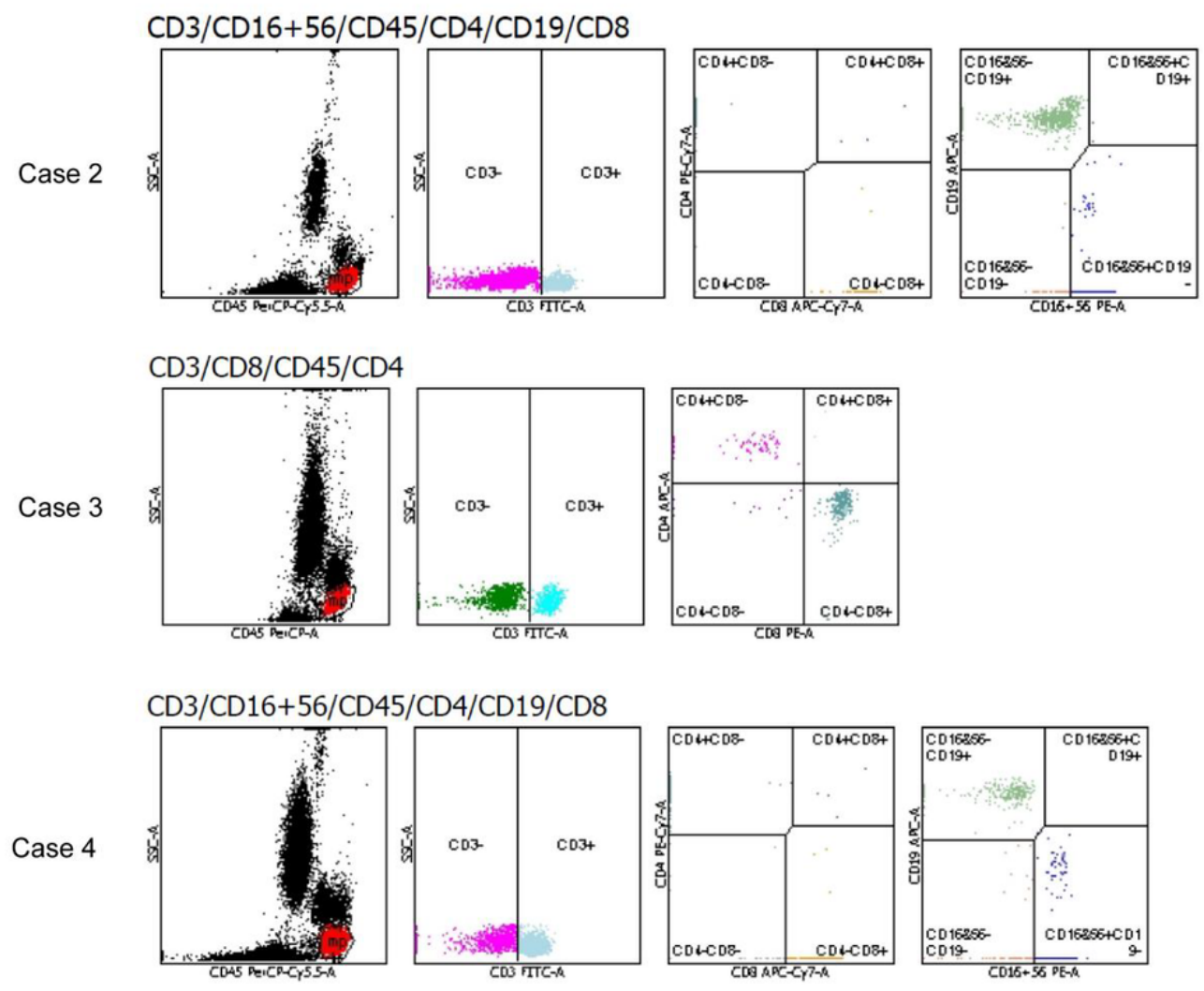

\section{Figure 1}

Clinical features A, Magnetic resonance images (MRI) of the upper abdomen in one patient (Case 2). Hepatomegaly and splenomegaly in cross-sectional, coronal and sagittal views, respectively. B, Flow cytometric assay for immunocytes of peripheral blood in the three patients with CAEBV infection was shown. Reduction of CD8+ T lymphocytes was seen in three cases. 
A

Ctrl

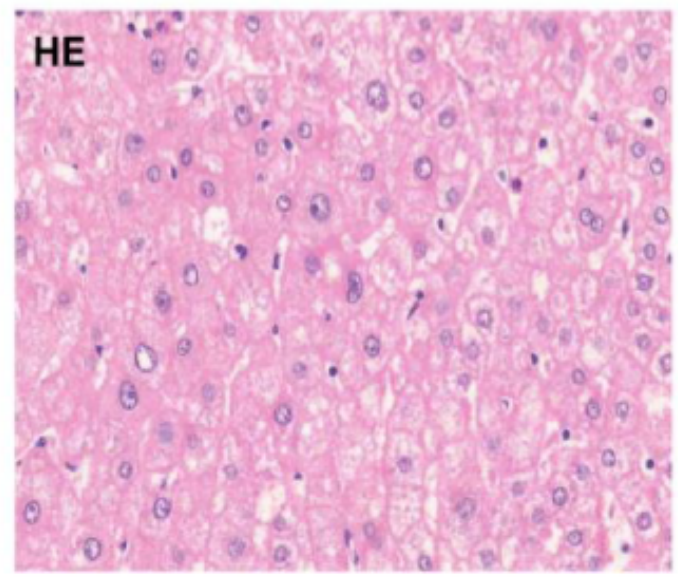

\section{CAEBV}

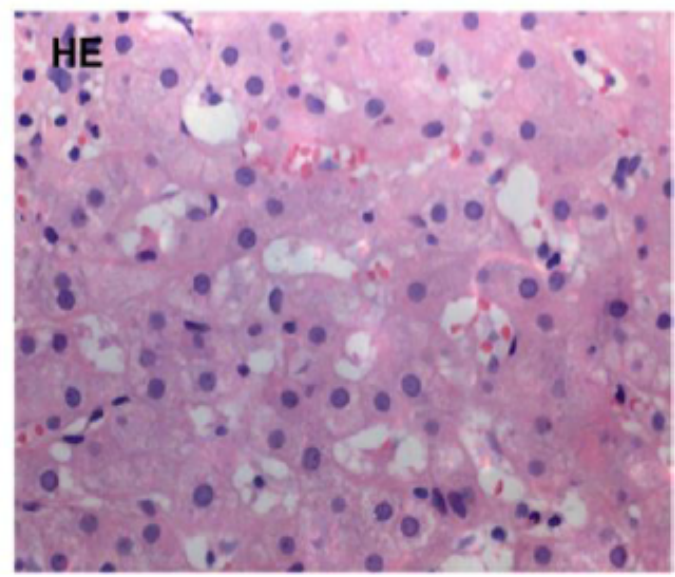

B

\section{CAEBV}
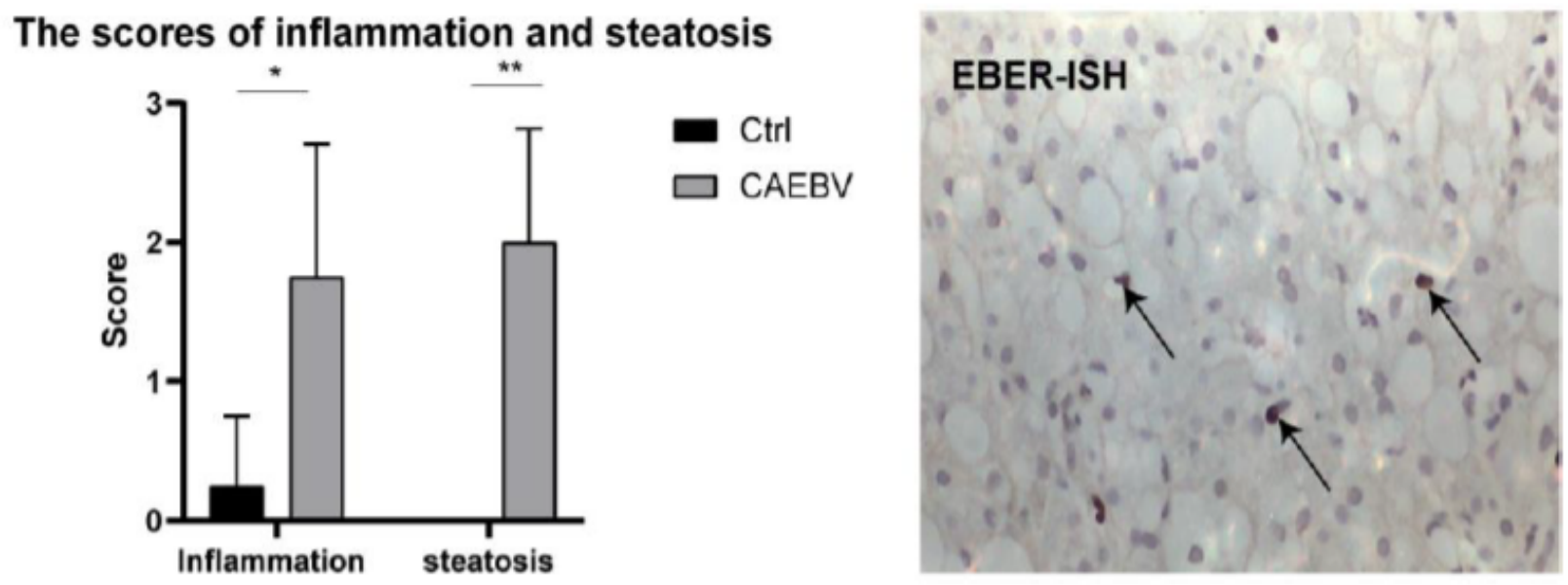

\section{Figure 2}

Morphologic Features and EBER-ISH in liver tissue A, HE staining of the control and CAEBVH groups were shown were shown. The liver tissue in patients with CAEBVH showed edematous degeneration and steatosis of hepatocytes. Lymphocytic infiltration in the hepatic sinusoids and confluent area in the CAEBVH group. B, Positive EBER-ISH in lymphocytes of patients with CAEBVH $(\times 200)$.

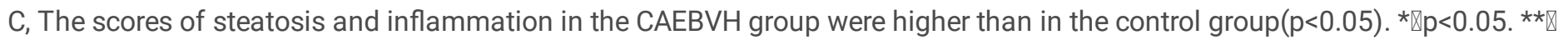
$p<0.01$. 


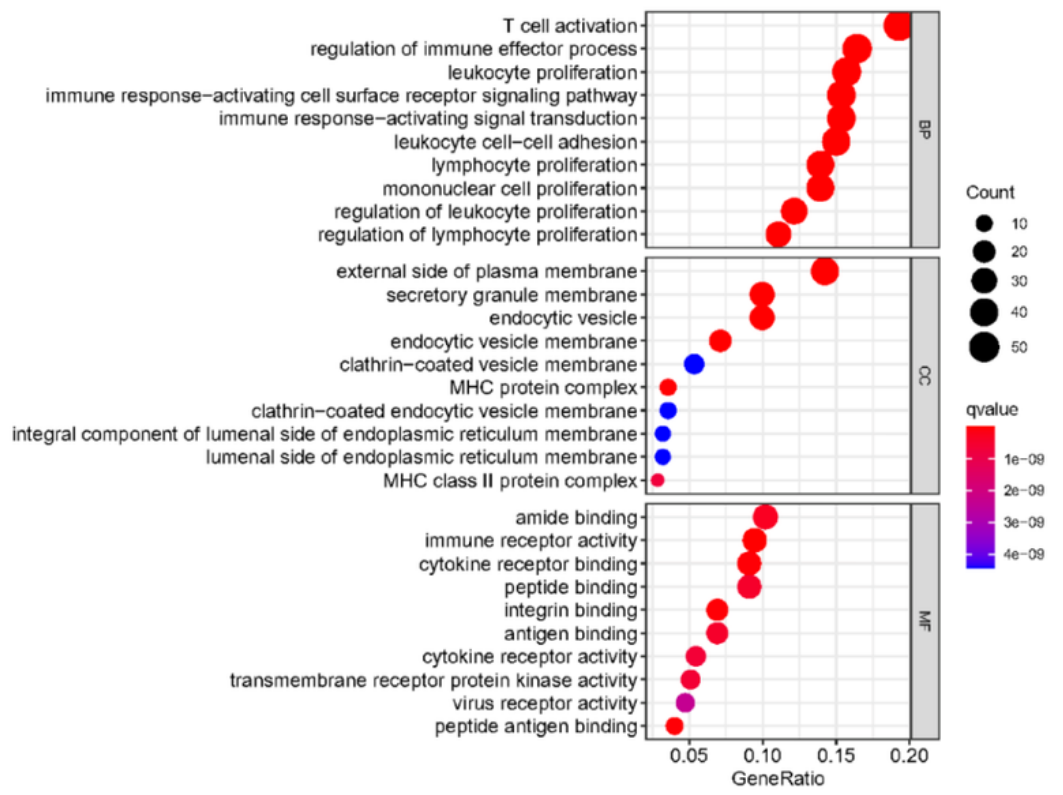

B

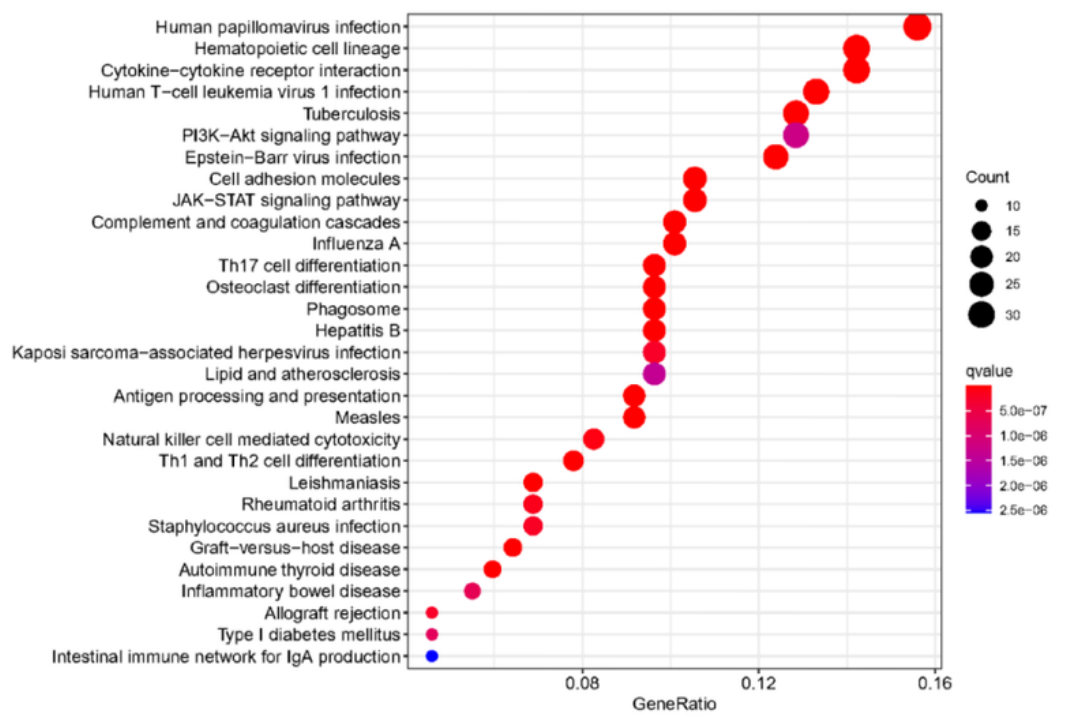

Figure 3

Mutant gene enrichment analysis A, GO enrichment analysis in the bubble chart was shown. B, KEGG enrichment analysis in the bubble chart was shown. The $\mathrm{X}$-axis indicates the gene ratio, and the $\mathrm{Y}$-axis indicates the pathway obtained by enrichment. The redder the color, the smaller the enriched q value; the more significant the dot, the more mutated genes in the corresponding pathway. 
Ctrl
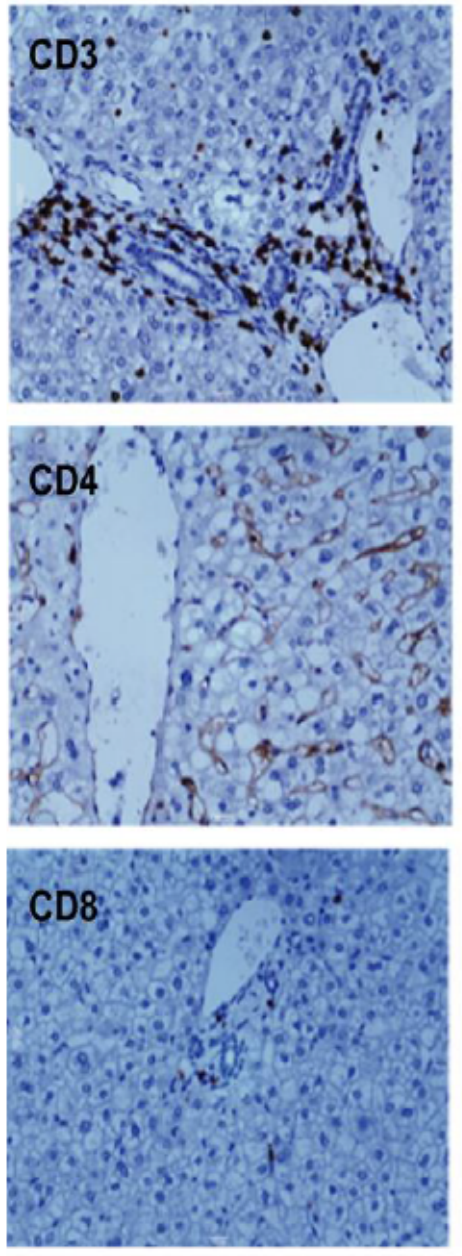

CAEBV
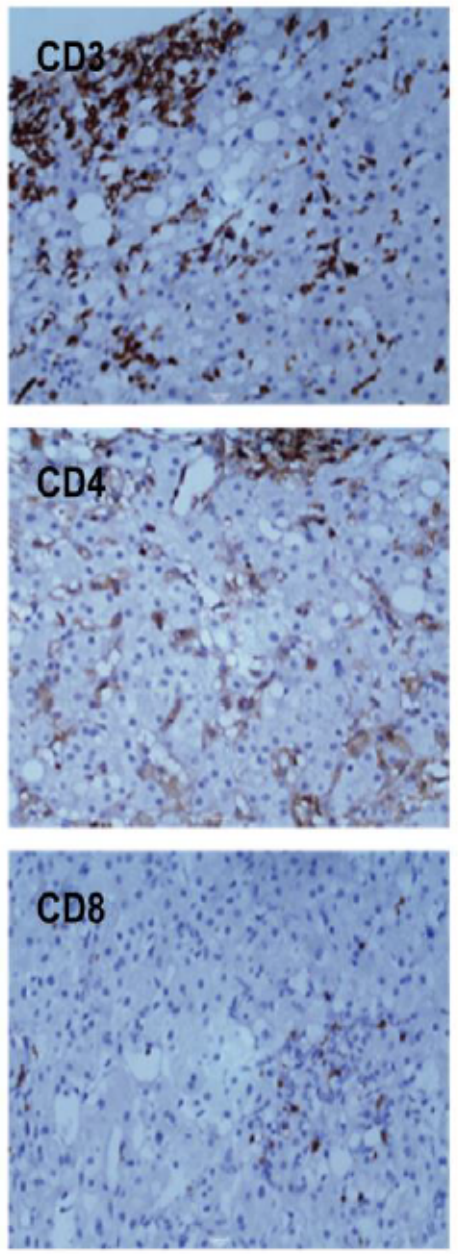

Ctrl
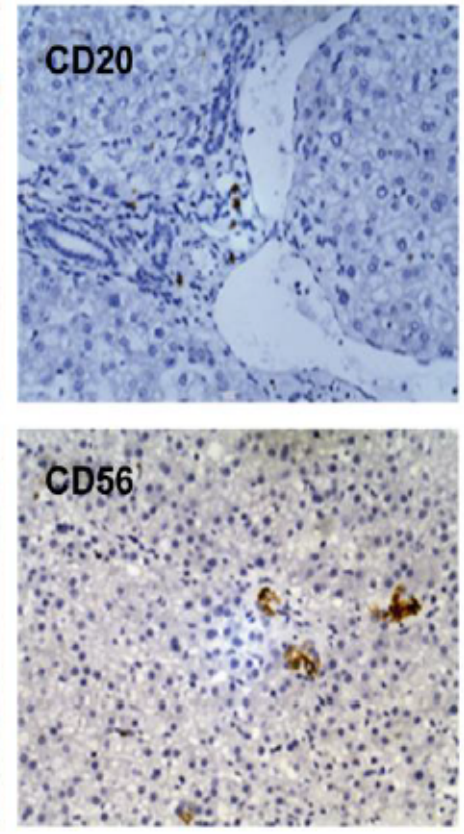

Immunocytes

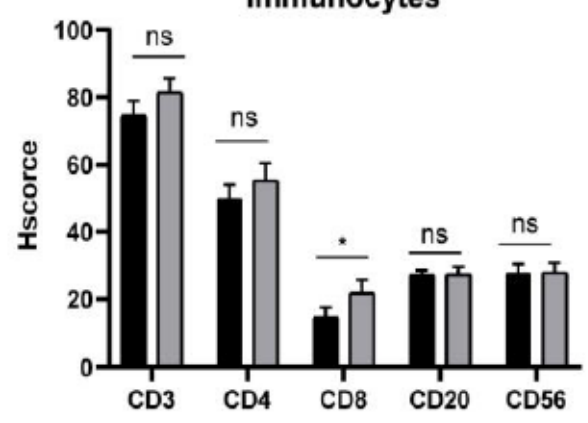

CAEBV
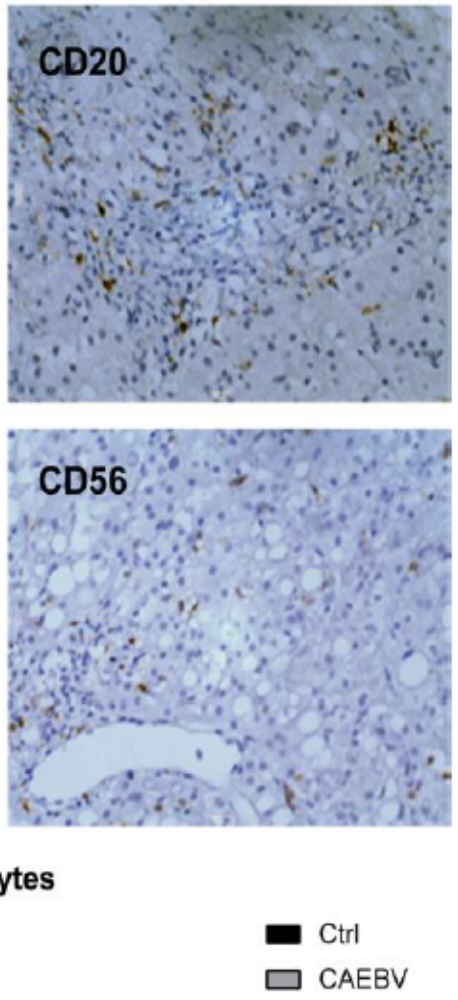

\section{Figure 4}

Immunophenotype of CAEBVH IHC staining of CD3, CD4, CD8, CD20 and CD56 in CAEBVH and control groups were shown ( $\times 200)$. The expression of CD8 was higher in the CAEBVH group than the control group $(p<0.05)$, but there were no significant differences in $C D 3, C D 4, C D 56$ and CD20 in the two groups ( $p>0.05)$. 
A

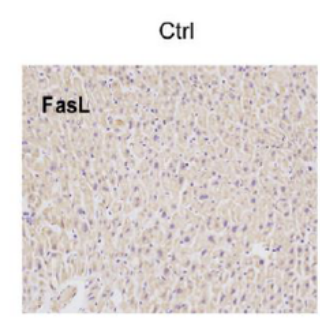

CAEBV
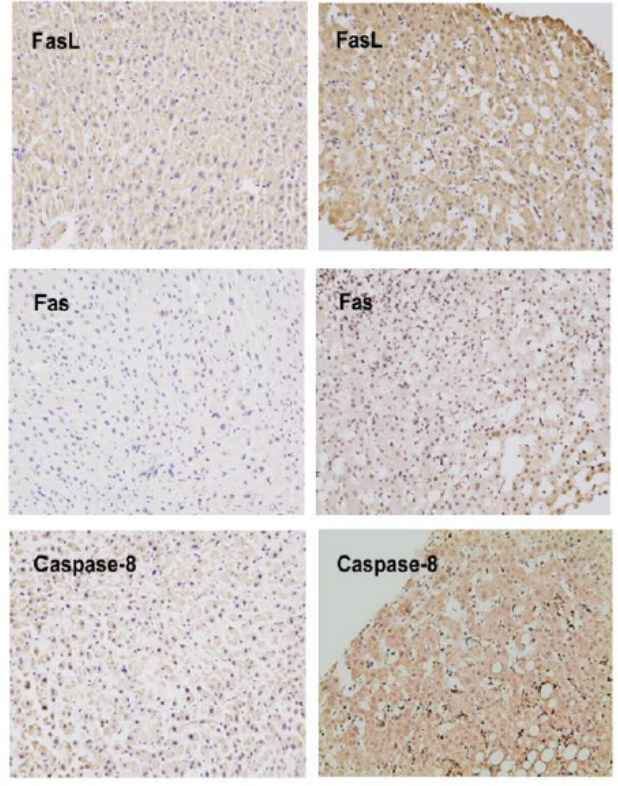

Ctrl
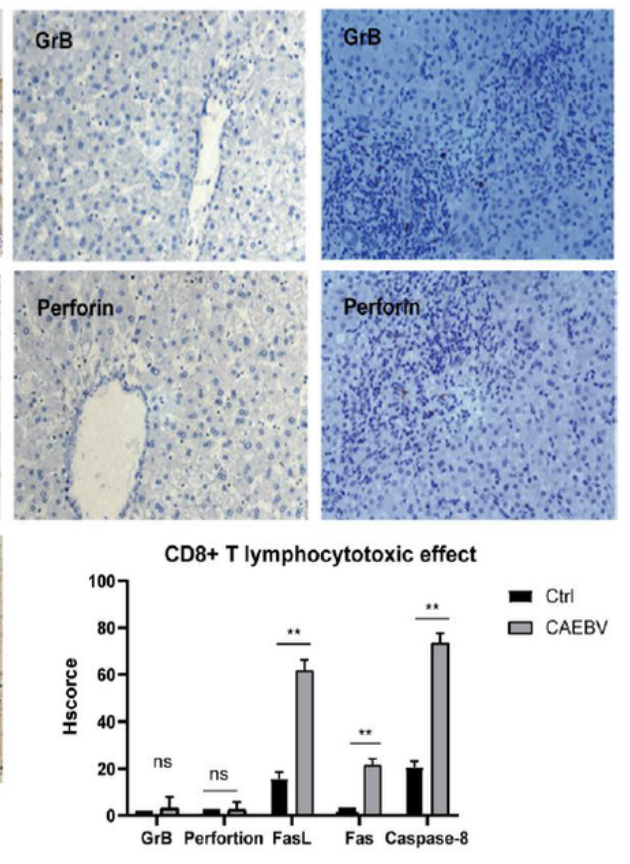

B
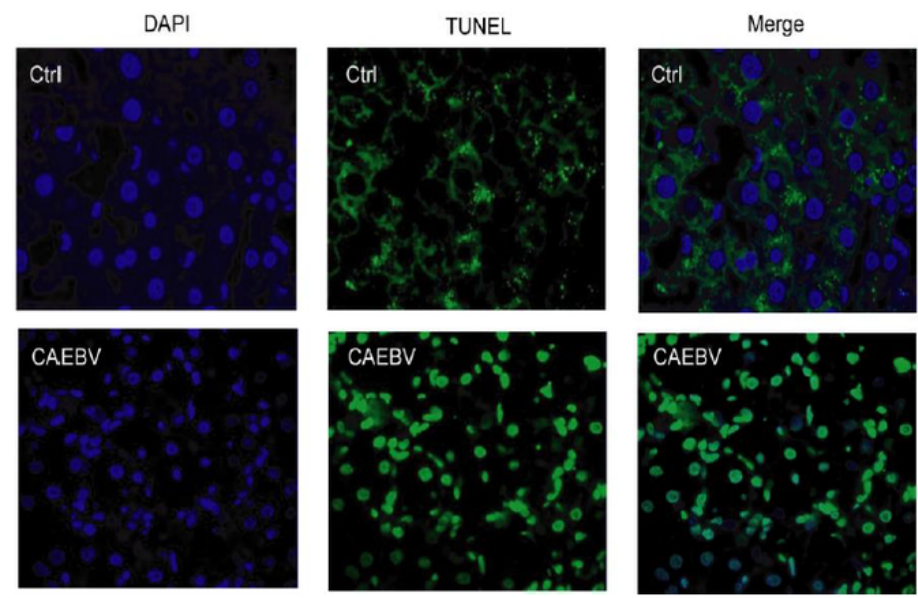

TUNEL

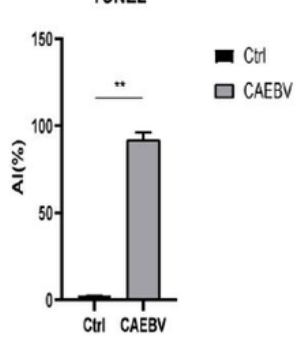

\section{Figure 5}

T-cell immune mechanism of CAEBVH A,IHC staining of GrB, perforin B, Fas, FasL and caspase-8 were shown $(\times 200)$. The expression of Fas, FasL and caspase-8 were higher in the CAEBVH group than the control group $(p<0.05)$, but the expression of $\mathrm{GrB}$ and perforin $\mathrm{B}$ were no significant differences $(\mathrm{p}>0.05)$. B, TUNEL detection of apoptosis CAEBV infection and the control group was shown. More cells apoptosis in the CAEBVH group than the control group $(p<0.05)$. 

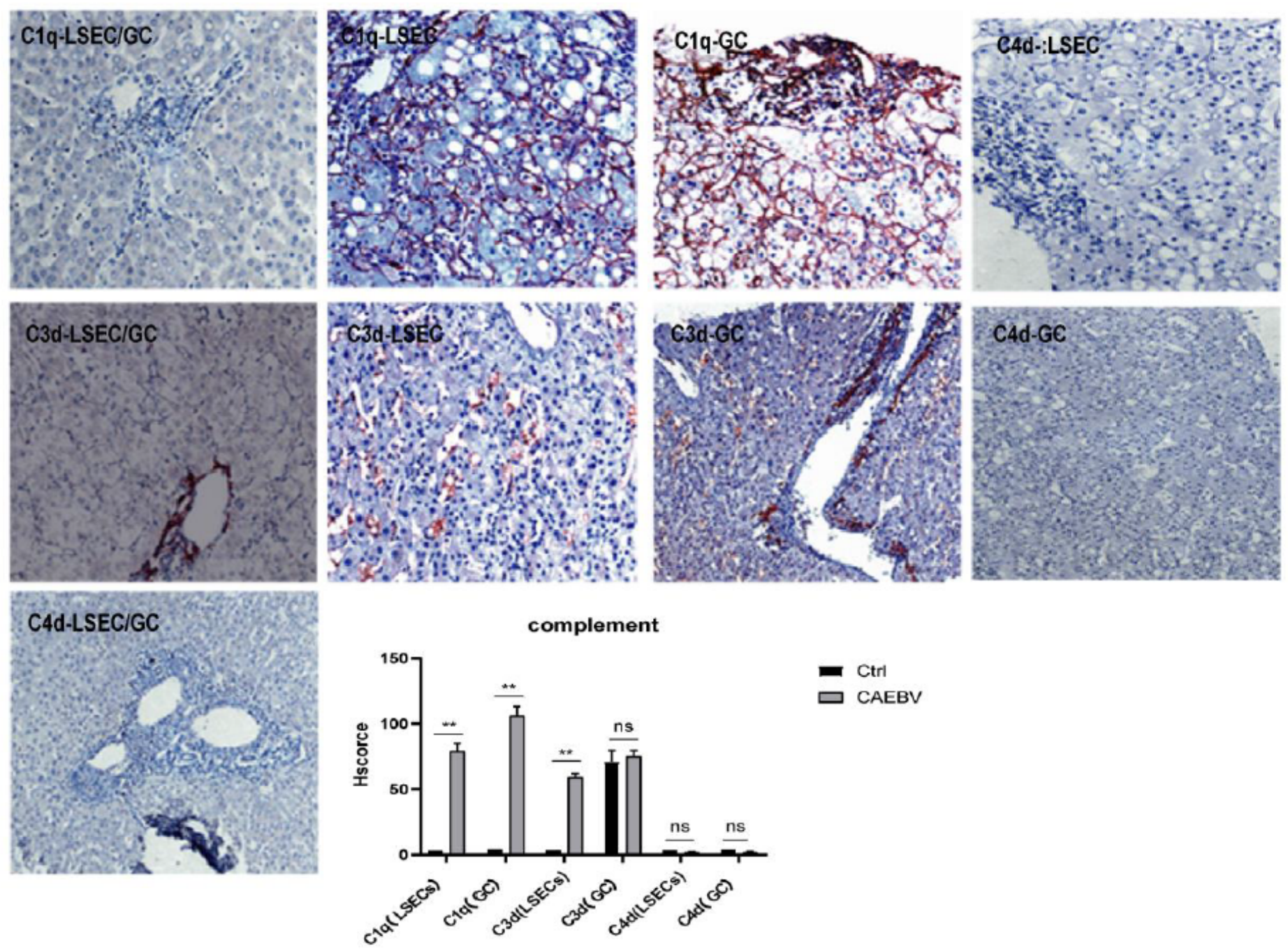

Figure 6

Complement activation of CAEBVH Expression of C1q, C3d and C4d in LESCs and GC of CAEBVH and control groups were shown. C1q expression of LESCs and GC as well as C3d expression of LESCs was higher in the CAEBVH group than the control group

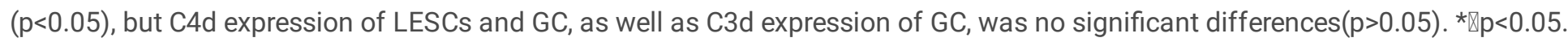
$* * \llbracket p<0.01$. 